\title{
Reversal of Azithromycin Resistance in Staphylococcus saprophyticus by Baicalin
}

\author{
Jinli Wang, Jinwu Meng, Jinyue Zhu, Tianxin Qiu, Wenjia Wang, Jinxue Ding, \\ Zhenguang Liu, Kun Li, Deyun Wang, Jiaguo Liu* and Yi Wu*
}

MOE Joint International Research Laboratory of Animal Health and Food Safety and Traditional Chinese Veterinary Medicine Research Center, College of Veterinary Medicine, Nanjing Agricultural University, Nanjing, China

OPEN ACCESS

Edited by:

Xin Zhao

McGill University, Canada

Reviewed by:

Francesca Paola Nocera,

Università degli Studi di Napoli

Federico II, Italy

Sougata Ghosh,

RK University, India

Jingui $L i$,

Yangzhou University, China

${ }^{*}$ Correspondence:

Jiaguo Liu

liujiaguo@njau.edu.cr

Yi Wu

wuyi2001cn@163.com

Specialty section

This article was submitted to Veterinary Infectious Diseases,

a section of the journal

Frontiers in Veterinary Science

Received: 02 December 2021

Accepted: 10 January 2022

Published: 18 February 2022

Citation:

Wang J, Meng J, Zhu J, Qiu T, Wang W, Ding J, Liu Z, Li K, Wang D, Liu J and Wu Y (2022) Reversal of Azithromycin Resistance in Staphylococcus saprophyticus by Baicalin. Front. Vet. Sci. 9:827674 doi: 10.3389/fvets.2022.827674
In recent years, the efficacy of antibiotics has been threatened by the evolution of bacterial resistance. We previously demonstrated that baicalin (Bac) showed synergies with azithromycin (Azm) against Azm-resistant Staphylococcus saprophyticus (ARSS). The aim of this study was to explore the roles of Bac in reversing the resistance of ARSS to Azm. The ARSS was sequentially passaged for 20 days with the sub-MIC (minimum inhibitory concentration) of Bac. The strain that recovered sensitivity to Azm was named Azm-sensitive S. saprophyticus (ASSS). The sub-MIC of Bac reversed the resistance of ARSS to Azm. The MIC of Azm against the ASSS strain was $0.488 \mathrm{mg} / \mathrm{l}$, and it was stable within 20 passages. The highest rate of resistance reversal reached 3.09\% after ARSS was exposed to $31.25 \mathrm{mg} / \mathrm{l} \mathrm{Bac}$ for 20 days. Furthermore, semiquantitative biofilm and RT-PCR assays reflected that the ability of biofilm formation and the transcript levels of $m s r A, m p h C$, and virulence-associated genes in the ASSS strain were significantly lower than those of the ARSS strain $(p<0.05)$. Simultaneously, Azm delayed the start time of death, alleviated the injury of the kidney, and decreased the bacterial burden in organs and cytokine levels in mice infected with ASSS. In contrast, Azm did not have a good therapeutic effect on mice infected with ARSS. Therefore, Bac has the potential to be an agent that reversed the resistance of ARSS to Azm.

Keywords: baicalin, S. saprophyticus, azithromycin, resistance, reversal

\section{INTRODUCTION}

Staphylococcus saprophyticus (S. saprophyticus), coagulase-negative coccus, causes urinary tract infections (1), francolin ophthalmia (2), and bovine mastitis (3). In poultry, macrolide antibiotics have often been used to treat infections induced with staphylococcus and streptococcus (4). It is reported that $60 \%$ of coagulase-negative staphylococci (CNS) isolated from various samples including central venous catheter tips, urine, and blood were highly resistant to penicillin (90\%), ceftriaxone (40\%), co-trimoxazole (60\%), and azithromycin (Azm) (60\%) (5). 
Many resistance genes of macrolides are parts of either transposon, plasmids, phages, or genomic islands and, as such, can easily transfer across species, strain, and genus boundaries (6). Researchers are increasingly identifying multidrug-resistant S. saprophyticus from ready-to-eat food of animal origin (7). The presence of resistance in food could be a severe threat to public health due to the possible spread of antibiotic resistance (7). However, resistance rates continue to rise, and the rate of antibiotic discovery has decreased substantially. In recent years, the combination of antibiotic drugs and non-antibiotics for bacterial infection treatment appears promising $(8,9)$. However, drug-resistant bacteria remain a severe threat to the efficacy of antibiotics. One strategy to deal with this problem was to recover the sensitivity of old antibiotics to bacteria. The methods of preventing the spread of antibiotic resistance need to be prioritized $(10,11)$.

Regulation of virulence factor production is essential for bacterial colonization and pathogenesis (12). Virulence inhibitors are important therapeutic means to treat bacterial infections (13). Virulence inhibitors only inhibit the colonization and virulence of bacteria without exerting selective pressure to hinder the emergence of antibiotic resistance (14). In S. saprophyticus, urease (ureC), Uro-adherence factor (UafA), autolysis Aas, surface-associated protein of S. saprophyticus (ssp), and Dserine deaminase (dsdA) are associated with colonization and pathogenicity $(1,15,16)$. The biofilm of $S$. saprophyticus could also cause persistent infection. Therefore, the development of effective virulence inhibitors is a potential method to deal with bacterial resistance.

Baicalin (Bac) is a potential candidate for reversing Azm resistance in Azm-resistant S. saprophyticus (ARSS), a flavonoid compound, extracted from Scutellaria baicalensis Georgi (17). It has been reported to restore the effectiveness of $\beta$-lactam antibiotics against $\beta$-lactam-resistant staphylococcus (17). We previously demonstrated that Bac combined with Azm exhibited synergistic activity against Azm-resistant S. saprophyticus (ARSS) (2). Although the synergy of Bac with Azm against ARSS has already been reported, its ability to reverse Azm resistance of ARSS and inhibit virulence has not previously been scrutinized.

Based on this information, we hypothesized that Bac had the potential to reverse the resistance of ARSS to Azm. Thus, the serial passage of ARSS exposure to the sub-MIC (minimum inhibitory concentration) of Bac was conducted. Moreover, the treatment efficacy of Azm to mice infected with the strain that recovered sensitivity to Azm by the serial passage was detected.

\section{MATERIALS AND METHODS}

\section{Strains and Culture Conditions}

Azm-resistant S. saprophyticus (Azm MIC of $1,000 \mathrm{mg} / \mathrm{l}$ determined by the broth micro-dilution method) was isolated from francolins suffering from ophthalmia in a francolin farm located in Jiangsu province, China (2). Furthermore, strains were

Abbreviations: ARSS, Azm-resistant S. saprophyticus; ASSS, Azm-sensitive S. saprophyticus; Azm, azithromycin; Bac, baicalin. cultured in Mueller-Hinton broth (MHB) or nutrient broth (NB, Hopebio, Qingdao, China) at $37^{\circ} \mathrm{C}$ with shaking at $180 \mathrm{rpm}$.

\section{The Rate of Resistance Reversal}

For the resistance reversal assay, $10^{6}$ colony-forming unit (CFU) exponential-phase ARSS was inoculated into $1 \mathrm{ml}$ of MHB containing Bac at sub-MICs: $250,125,62.5,31.25 \mathrm{mg} / \mathrm{l}$. After $24 \mathrm{~h}$, the cultures allowed growth were further diluted at 1:100 with fresh MHB containing Bac. The passaging process was repeated for 20 days consecutively. Every five passages, approximately $1 \times 10^{3} \mathrm{CFU}$ ARSS strain exposed to sub-MIC levels of Bac were cultured on mannitol salt agar (MSA) plates at $37^{\circ} \mathrm{C}$. After $18 \mathrm{~h}$ of incubation, the colonies from the MSA plate were transferred onto a drug-free MSA plate and an MSA plate containing $250 \mathrm{mg} / \mathrm{l} \mathrm{Azm}$. Then the plates were cultured for $24 \mathrm{~h}$ at $37^{\circ} \mathrm{C}$. The numbers of colonies surviving in the plate containing Azm and drug-free plate were respectively recorded as $\mathrm{C}_{1}$ and $\mathrm{C}_{2}$. Finally, the rate of resistance reversal was calculated based on the formula: resistance reversal rate $(\%)=\left(1-\mathrm{C}_{1} / \mathrm{C}_{2}\right)$ $\times 100 \%$. The strains that were recovered sensitivity to Azm were named Azm-sensitive S. saprophyticus (ASSS).

\section{MIC Determination}

The ASSS and ARSS strains in the logarithmic growth phase were diluted to a final concentration of $1 \times 10^{6} \mathrm{CFU} / \mathrm{ml}$ in MHB. Azm and Bac were diluted using a two-fold serial dilution to obtain the target concentration with MHB in a 96-well plate. A total of $50 \mu \mathrm{l}$ of bacteria suspension was mixed with $50 \mu \mathrm{l}$ of the compound and incubated aerobically for $18-24 \mathrm{~h}$ at $37^{\circ} \mathrm{C}$. The lowest concentration which inhibited the visible growth of bacteria was defined as the MIC value. All of the assays were performed in triplicate independently with two samples.

\section{The Stability of Resistance Reversal}

The ASSS and ARSS strains were passaged in MHB without Bac. Briefly, exponential-phase bacteria $\left(10^{6} \mathrm{CFU} / \mathrm{ml}\right)$ were grown in MHB and cultured for $24 \mathrm{~h}$. Then, the cultures were diluted at 1:100 into fresh MHB. The serial passages were conducted daily until the 20th passage. At least three independent biological replicates of each experiment were carried out. The MIC was determined as described as above every five passages.

\section{Determination of Growth Curve}

For the growth kinetic assay of ARSS and ASSS, overnight cultures were prepared and cultured in NB. The cell densities at $600 \mathrm{~nm}$ were measured at $0,2,4,6,8,10,12$, and $24 \mathrm{~h}$. For the growth curve under Bac, overnight ARSS cultures were prepared and cultured in MHB containing sub-MIC of Bac. The cell densities at $600 \mathrm{~nm}$ were measured at $0,2,4,6,8,10,12,14$, and $24 \mathrm{~h}$.

\section{Gene Transcription Levels Measured by RT-PCR}

To measure the mRNA transcript levels of $m s r A, m p h C$, $d s d A$, ure C, Aas, UafA, and ssp genes, RT-PCR was conducted as previously described (18). The Bacteria RNA Extraction Kit (Angle Gene, Nanjing, China) was used to extract the total 
RNA from bacterial cells in the mid-log phase. The values of $A_{260} / A_{280}$ were $1.8-2.1$. The RNA was used to reverse into cDNA using a HiScript II 1st Strand cDNA Synthesis Kit (Vazyme, Nanjing, China). Thereafter, the reverse transcription was performed at $50^{\circ} \mathrm{C}$ for $15 \mathrm{~min}$ and $85^{\circ} \mathrm{C}$ for $5 \mathrm{~s}$. RT-PCR reactions were carried out in a StepOne PCR instrument (Applied Biosystems, Foster City, CA, USA) using ChamQ ${ }^{\mathrm{TM}} \mathrm{SYBR}^{\circledR}$ qPCR Master Mix (Vazyme, Nanjing, China) as recommended by the manufacturer's instructions. The protocol of the RT-PCR reaction was as follows: first holding stage at $95^{\circ} \mathrm{C}$ for $3 \mathrm{~min}$, followed by a cycling stage at $95^{\circ} \mathrm{C}$ for $10 \mathrm{~s}$ and $60^{\circ} \mathrm{C}$ for $60 \mathrm{~s}$ (40 cycles total), and a final melting curve stage at $95^{\circ} \mathrm{C}$ for $15 \mathrm{~s}$, then $60^{\circ} \mathrm{C}$ for $60 \mathrm{~s}$, and $95^{\circ} \mathrm{C}$ for $15 \mathrm{~s}$. The housekeeping gene $16 S r R N A$ was chosen as the internal control gene. The method of $2^{-\Delta \Delta C T}$ was used to analyze the data which were presented relative to the ARSS group. Primer sequences used are shown in Table 1.

\section{Biofilm Assay}

Biofilm formation assays were performed following a previously published protocol with slight modifications (19). Briefly, a 96-well plate was inoculated with $1 \times 10^{5} \mathrm{CFU} / \mathrm{ml}$ ARSS or ASSS suspension $(200 \mu \mathrm{l} /$ well $)$ and incubated at $37^{\circ} \mathrm{C}$ without shaking. After $24 \mathrm{~h}$, the supernatants were removed, and wells were washed with PBS twice. Afterward, the attached bacteria were fixed by $2.5 \%$ glutaraldehyde for $1.5 \mathrm{~h}$ and then air-dried at room temperature. The wells were stained by adding $200 \mu 1$ 1\% (wt/vol) crystal violet for $20 \mathrm{~min}$ and then rinsed thoroughly with PBS until the negative control wells (without bacteria) became colorless. Finally, $200 \mu \mathrm{l}$ of $33 \%$ glacial acetic acid was added to the wells and the absorbance values were detected at $570 \mathrm{~nm}$ using a Thermo ${ }^{\mathrm{TM}}$ Multiskan $^{\mathrm{TM}} \mathrm{FC}$ enzyme-labeled instrument.

TABLE 1 | Oligonucleotide primers used in this study.

\begin{tabular}{|c|c|c|c|}
\hline $\begin{array}{l}\text { Target } \\
\text { gene }\end{array}$ & Primer & Sequence $\left(5^{\prime}-3^{\prime}\right)$ & Source \\
\hline \multirow[t]{2}{*}{$16 S$ rRNA } & $16 S$ rRNA-F & AGTTGTTCTCAGTTCGGATT & This study \\
\hline & $16 S$ rRNA-R & ATACGGCTACCTTGTTACG & \\
\hline \multirow[t]{2}{*}{$m s r A$} & $m s r A-F$ & GCTCTACTGAATGATTCTGATG & This study \\
\hline & $m s r A-R$ & TGGCATACTATCGTCAACTT & \\
\hline \multirow[t]{2}{*}{ mphC } & $m p h C-F$ & GAGACTACCAAGAAGACCTGACG & This study \\
\hline & $m p h C-R$ & CATACGCCGATTCTCCTGAT & \\
\hline \multirow[t]{2}{*}{$d s d A$} & $d s d A-F$ & GTGGAAGTCATAGAACATCAG & This study \\
\hline & $d s d A-\mathrm{R}$ & GCGTCATCATACCTAATAGC & \\
\hline \multirow[t]{2}{*}{ ureC } & ureC-F & ACACATATCGGTGGCGGTACAG & This study \\
\hline & ureC-R & GGTTACAGCTTGCCCTTACCAG & \\
\hline \multirow[t]{2}{*}{ Aas } & Aas-F & GCCGACTACGCAGCAACTAAC & This study \\
\hline & Aas- $\mathrm{R}$ & CCATGAGGGTCAGAGTGGTCAG & \\
\hline \multirow[t]{2}{*}{ uafA } & uafA-F & TTCGGTGGTTATGTATGGTT & This study \\
\hline & uafA-R & CAGTGTTGTTCGCTTGTG & \\
\hline \multirow[t]{2}{*}{$s s p$} & $s s p-F$ & ACTTCGGTCTATCTCAATGG & This study \\
\hline & $s s p-R$ & ACATCTGTTGCTTCGGTATA & \\
\hline
\end{tabular}

The data were calculated by subtracting the values in the negative control from all the experimental groups.

\section{Mouse Model of ASSS or ARSS Infection}

For the experiment, 100 female ICR mice (18-20 g) were randomized into five groups: ASSS group, ASSS-Azm group, ARSS group, ARSS-Azm group, and blank control (BC) group. For mice in the ASSS and ASSS-Azm groups, they were intraperitoneally injected with $3.6 \times 10^{9} \mathrm{CFU}$ of the ASSS strain in sterile normal saline. The mice in the ARSS and ARSS-Azm group were challenged with $2 \times 10^{9} \mathrm{CFU}$ of the ARSS strain in sterile normal saline. In the $\mathrm{BC}$ group, mice were incubated with equal-volume normal saline. Two hours later, mice in the ASSS-Azm and ARSS-Azm groups were orally administrated with Azm at a dosage of $75 \mathrm{mg} / \mathrm{kg}$, once a day for 3 days. To ensure consistency across tests, mice in the other groups were treated with equal-volume normal saline. At $10 \mathrm{~h}$ postinfection, five mice were anesthetized and blood was collected via retroorbital bleeding. Then, clotted blood was centrifuged at 3,000 rpm for $15 \mathrm{~min}$ and serum was collected. The levels of IL-6, IL8 , and TNF- $\alpha$ in serum were measured with ELISA kits (Angle Gene, Nanjing, China). The liver, spleen, kidney, and bladder were removed after thoracotomy, weighed, and homogenized in sterile saline solution. The serially diluted samples were plated onto MSA plates for CFU enumeration. For hematoxylin and eosin (H\&E) staining and examination, the kidney was collected and fixed in $4 \%$ paraformaldehyde. The mice were monitored for a survival rate up to $58 \mathrm{~h}$.

\section{Statistical Analysis}

Mean \pm standard deviation (SD) was used to express the data. Duncan's multiple-range tests and independent-sample tests were performed to analyze the data among groups using SPSS software (IBM SPSS Statistics 20.0). The statistical significance level was $P$-value $\leq 0.05$.

\section{RESULTS}

\section{The Growth Curve of ARSS Under sub-MIC of Bac}

Previously, we indicated that the MIC of Bac against ARSS was $500 \mathrm{mg} / \mathrm{l}$ (2). In this experiment, the growth curves of ARSS under sub-MIC of Bac were measured. As shown in Figure 1, $250 \mathrm{mg} / \mathrm{l} \mathrm{Bac}$ inhibited the growth of ARSS in MHB. The start time of the exponential phase of the $250-\mathrm{mg} / \mathrm{l} \mathrm{Bac}$ group was $2 \mathrm{~h}$ later than the other groups. In the 250-mg/l Bac group, the $\mathrm{OD}_{600 \mathrm{~nm}}$ values were three-fold lower than those of the control group at $12 \mathrm{~h} .125$ and $62.5 \mathrm{mg} / \mathrm{l} \mathrm{Bac}$ moderately inhibited the growth of ARSS. However, we can detect a marked multiplication of strain in the $31.25-\mathrm{mg} / \mathrm{l} \mathrm{Bac}$ and untreated groups.

\section{The Effect of Bac Reversed the Resistance of ARSS to Azm}

As shown in Figure 2A, the rates of resistance reversal gradually increased in the presence of sub-MIC of Bac over time. Importantly, in the 20th passage, the rate of resistance reversal was the highest, reaching $3.09 \%$ in the $31.25-\mathrm{mg} / \mathrm{l}$ Bac group. The 


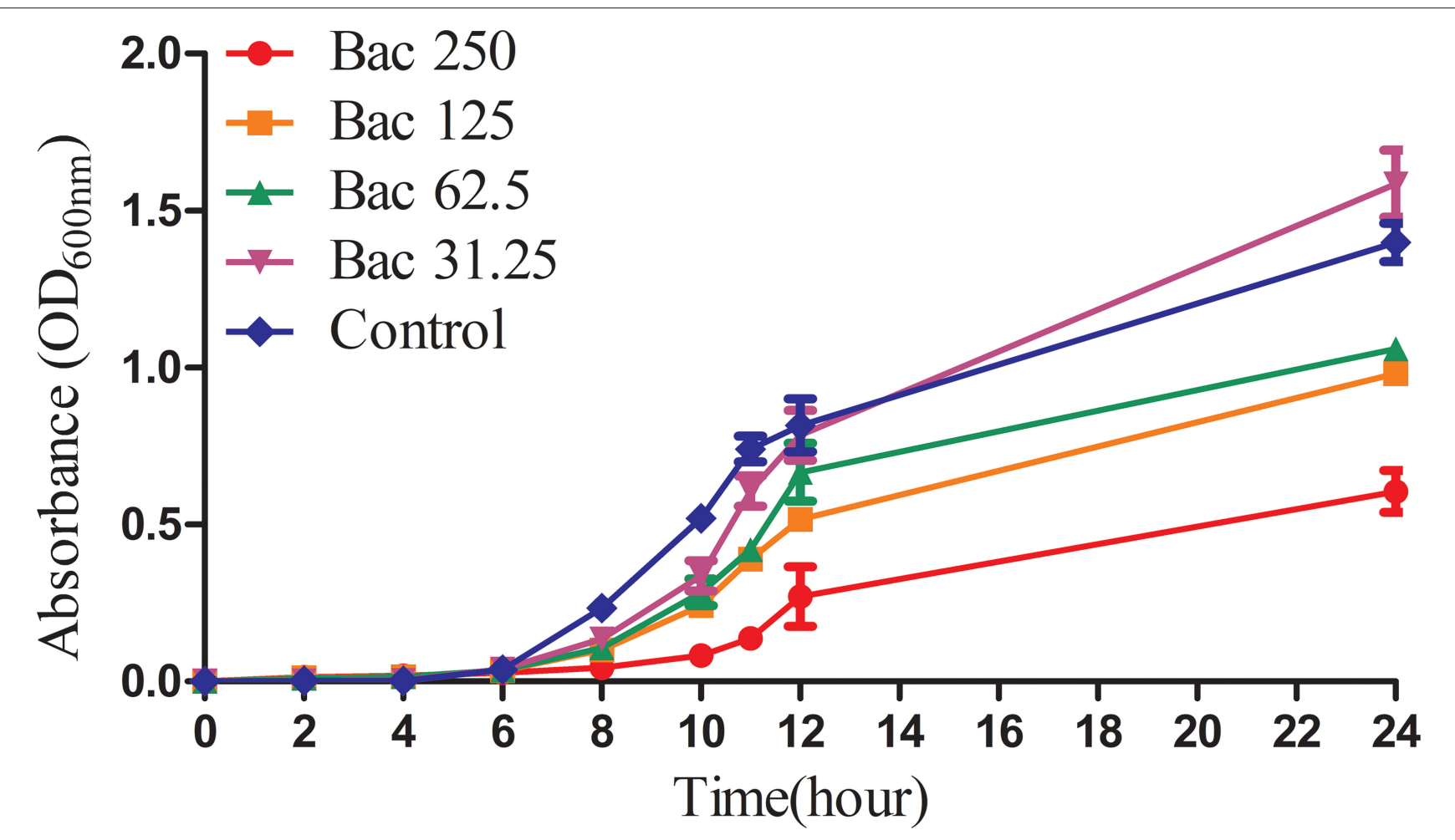

FIGURE 1 | The growth curves of ARSS in MHB under the sub-inhibitory concentration of Bac. $\mathrm{OD}_{600 \mathrm{~nm}}$ values were the means \pm SD from three independent experiments.

strain that recovered sensitivity to Azm was named ASSS. Then, the MIC and stability of resistance reversal were determined. The MIC of Azm against ASSS decreased 2,049-fold to 0.488 mg/l compared with the ARSS strain. The MICs of Bac against ARSS and ASSS were $500 \mathrm{mg} / \mathrm{l}$ (Table 2), and the MICs of Azm against ASSS and ARSS were stable within 20 passages in nondrug MHB (Figure 2B). Simultaneously, the growth curves of ARSS and ASSS were similar in NB (Figure 2C).

\section{The Changes of the Transcript Levels of Resistance-Associated Genes and Biofilm Formation Ability}

To measure the mRNA transcript levels of resistance-associated genes in ARSS and ASSS, RT-PCR was performed. As shown in Figure 3A, the mRNA transcript levels of $m s r A$ and $m p h C$ genes in the ASSS group were prominently lower than those in the ARSS group $(P<0.05)$. Biofilm also confers resistance to antibiotics. Therefore, the ability of biofilm formation was measured in ARSS and ASSS. The $A_{570}$ values of the ASSS group were remarkably reduced with a 2 -fold decrease compared to the ARSS group (Figure 3B, $P<0.05$ ).

\section{The mRNA Transcript Levels of Virulence Genes}

To investigate the changes of the mRNA transcript levels of virulence genes, RT-PCR was carried out. Compared to the ARSS
TABLE 2 | Minimal inhibitory concentrations (mg/L) of Azm and Bac.

\begin{tabular}{lll}
\hline Strain & \multicolumn{2}{c}{ MIC (mg/L) } \\
\cline { 2 - 3 } & Azm & Bac \\
\hline ARSS & 1,000 & 500 \\
ASSS & 0.488 & 500
\end{tabular}

Azm, azithromycin; Bac, baicalin.

strain, the transcript levels of ure C, Aas, uafA, and ssp genes of the ASSS strain significantly degraded $(P<0.05)$. However, the RT-PCR data of $d s d A$ gene manifested that no pronounced differences were observed between the ARSS and ASSS groups (Figure 4, $P>0.05$ ).

\section{The Clinical Curative Effect of Azm to ASSS or ARSS-Associated Infection}

The clinical curative effect of Azm is presented in Figure 5. In the ASSS group, infection with the ASSS strain resulted in rapid mouse death, with a $0 \%$ survival rate occurring within $25 \mathrm{~h}$. However, in the ASSS-Azm group, the first death occurred at $14 \mathrm{~h}$ postinfection, and approximately $6.7 \%$ of mice remained alive at $58 \mathrm{~h}$. However, the survival rate of the ARSS-Azm group (33.3\%) was similar to that of the ARSS group (26.7\%). 


\section{A}

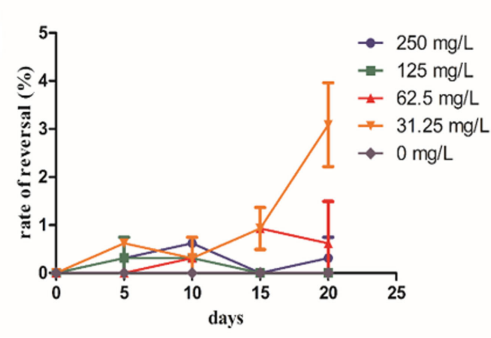

B

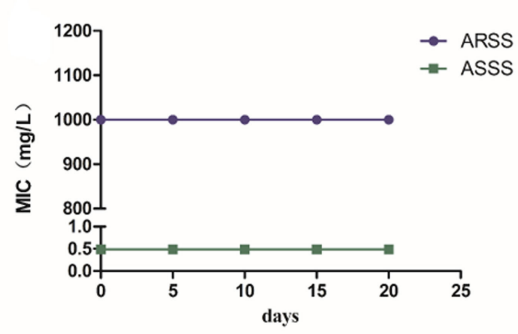

C

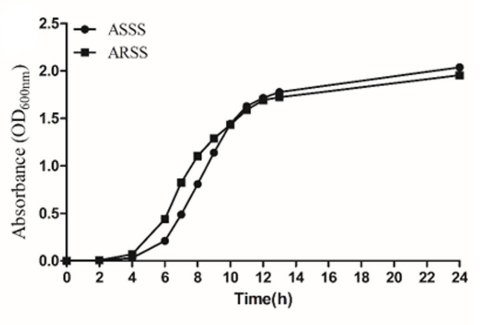

FIGURE 2 | Bac reversed the resistance of ARSS to Azm. (A) Rate of resistance reversal development of ARSS to Azm during serial passaging in the presence of sub-inhibitory concentrations of Bac. (B) The MICs of Azm against ARSS and ASSS during passaging under non-drug MHB. (C) The growth curve of ARSS and ASSS. $\mathrm{OD}_{600 \mathrm{~nm}}$ values are the means $\pm \mathrm{SD}$. All tests were done with three biological replicates.

A

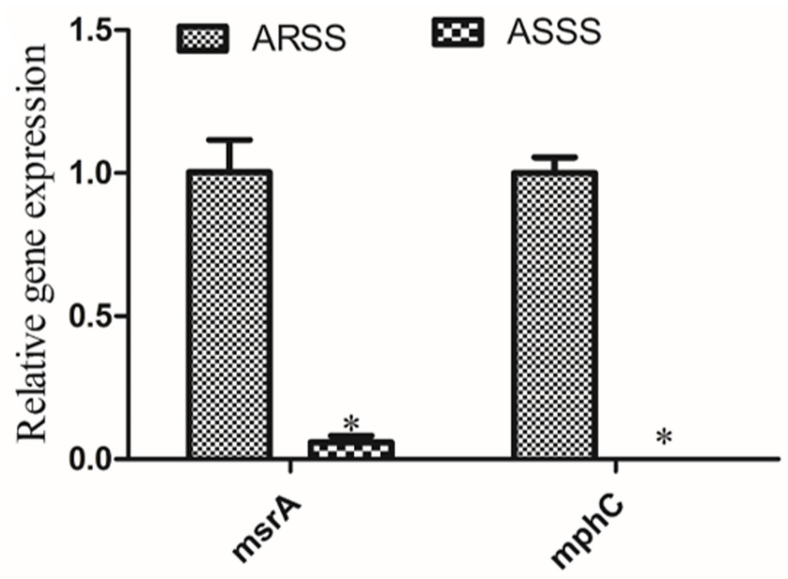

B

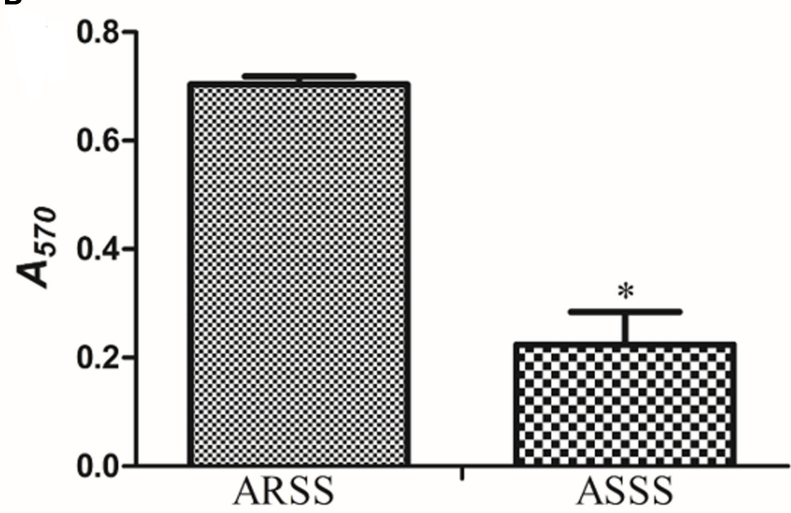

FIGURE 3 | The changes of the transcript levels of resistance-associated genes and biofilm formation ability. (A) The transcript levels of $m s r A$ and $m p h C$ genes were detected by the RT-PCR method. (B) The biomass of ARSS and ASSS was assessed by the crystal violet assay. ${ }^{*} p<0.05$.

\section{The Changes of Bacterial Burdens}

In order to explore the influences of Azm during ASSS or ARSS infection, the bacterial burdens were calculated in the liver, spleen, kidney, and bladder. Post $8 \mathrm{~h}$ infection, compared to the ASSS group, the proliferation level of the ASSS strain in the ASSS-Azm group was significantly reduced (Figures 6A-D). Additionally, the bacterial distribution data showed that no statistically obvious difference was found between the ARSS and ARSS-Azm groups (Figures 6E-H). These results revealed that Azm efficiently inhibited the proliferation of the ASSS strain in the tissues rather than the ARSS strain.

\section{Pathological Changes of the Kidney}

The kidney histological changes of each group observed by HE staining are summarized in Figure 7. As shown in the figures, no lesion was observed in the kidney of the BC group. However, the histopathology of the mice in other groups showed that atrophic glomerulus (indicated by arrows) was observed at $8 \mathrm{~h}$ postinfection. Furthermore, kidney histological changes of the ASSS-Azm group were significantly alleviated with an obvious decrease in the atrophic glomerulus. In contrast, the histological changes of the ARSS-Azm group were similar to those of the ARSS group.

\section{Serum Cytokine Levels}

ELISA was carried out to detect the levels of IL-6, IL-8, and TNF$\alpha$ in serum at $8 \mathrm{~h}$ postinfection. As illustrated in Figure 8, in the BC group, the levels of IL-6, IL- 8 , and TNF- $\alpha$ were remarkably lower than those in the other groups $(P<0.05)$. Compared with the ASSS group, Azm significantly induced a decrease of IL-6, IL8 , and TNF- $\alpha$ in the ASSS-Azm group $(P<0.05)$. In addition, the results of cytokine levels indicated that there were no pronounced differences between ARSS-Azm and ARSS groups (Figure 8).

\section{DISCUSSION}

Global healthcare was threatened by the rapid emergence and spread of multidrug-resistant bacteria. Coupled with the fact that the development of novel antibiotics is very slow, it is necessary to develop antibiotic adjuvants to reverse antibiotic resistance (9). Bacteria that are previously sensitive to commonly used antibiotics became resistant by stepwise 


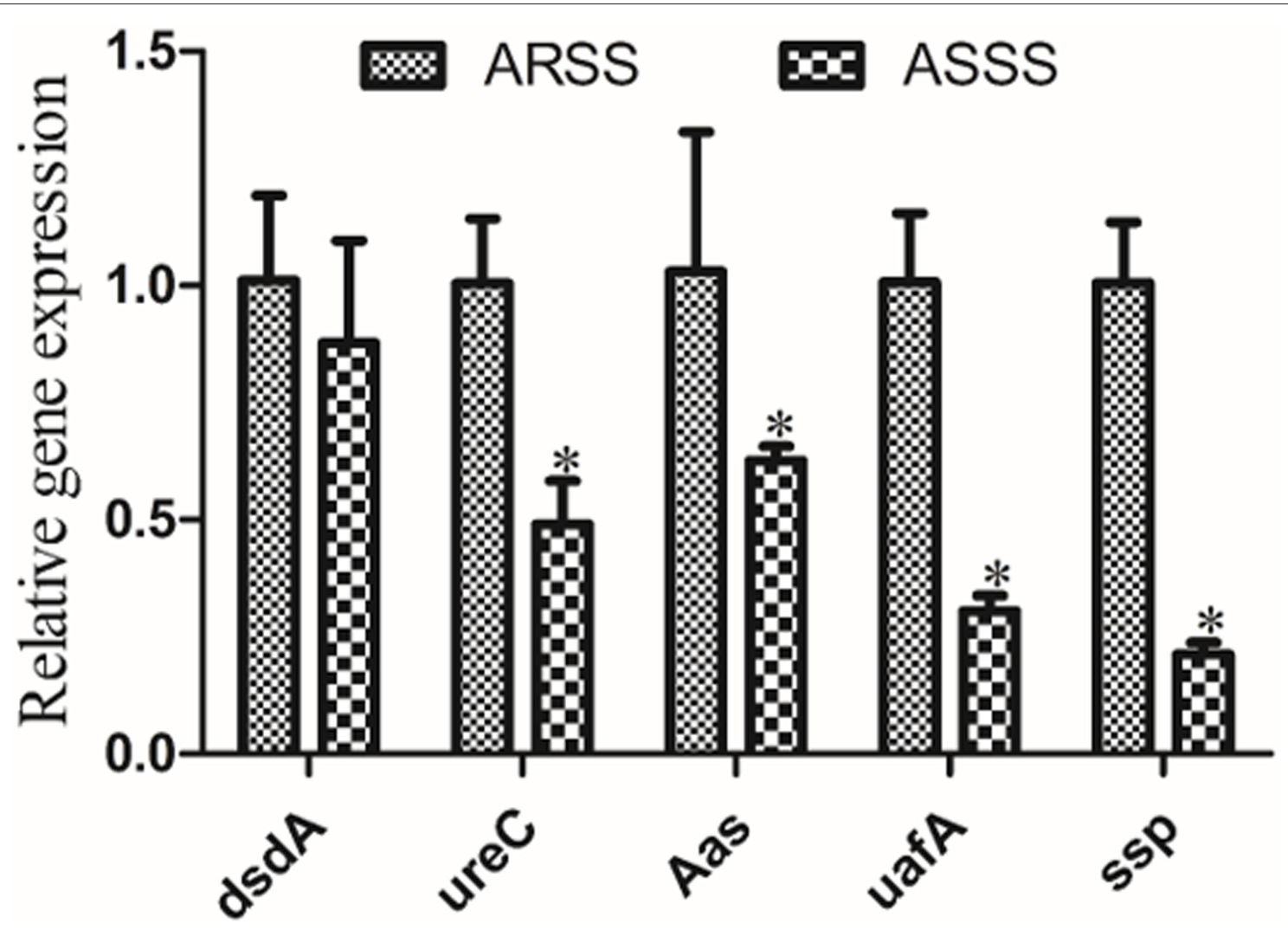

FIGURE 4 | RT-PCR measurement of the differentially virulence-associated genes in the ARSS and ASSS strains. Five genes, i.e., dsdA, ureC, Aas, uafA, and ssp, were selected and amplified using RT-PCR. The 16S rRNA gene was used as the internal control. Significant differences are marked by asterisks ( $\left.{ }^{*} p<0.05\right)$.

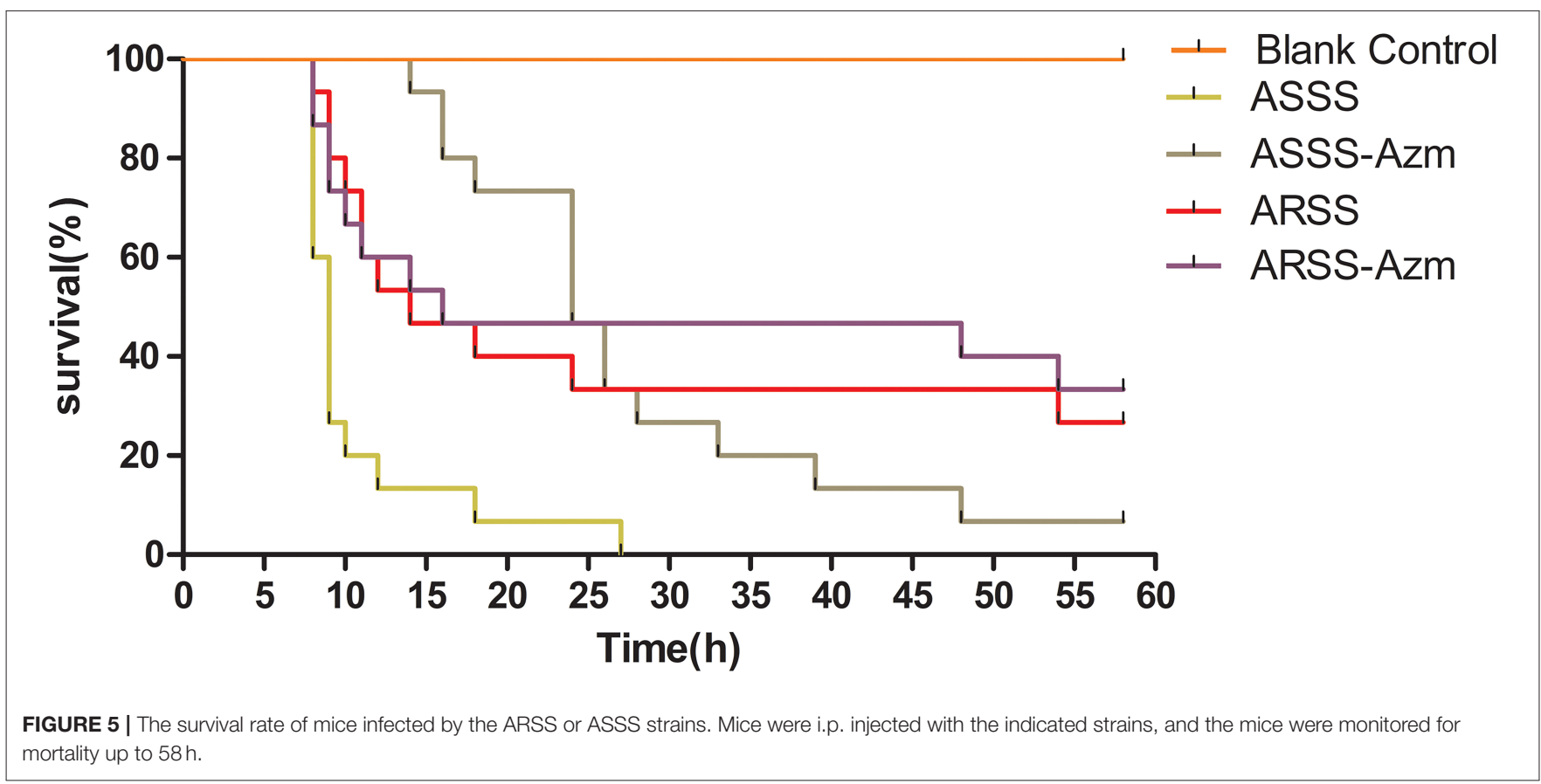



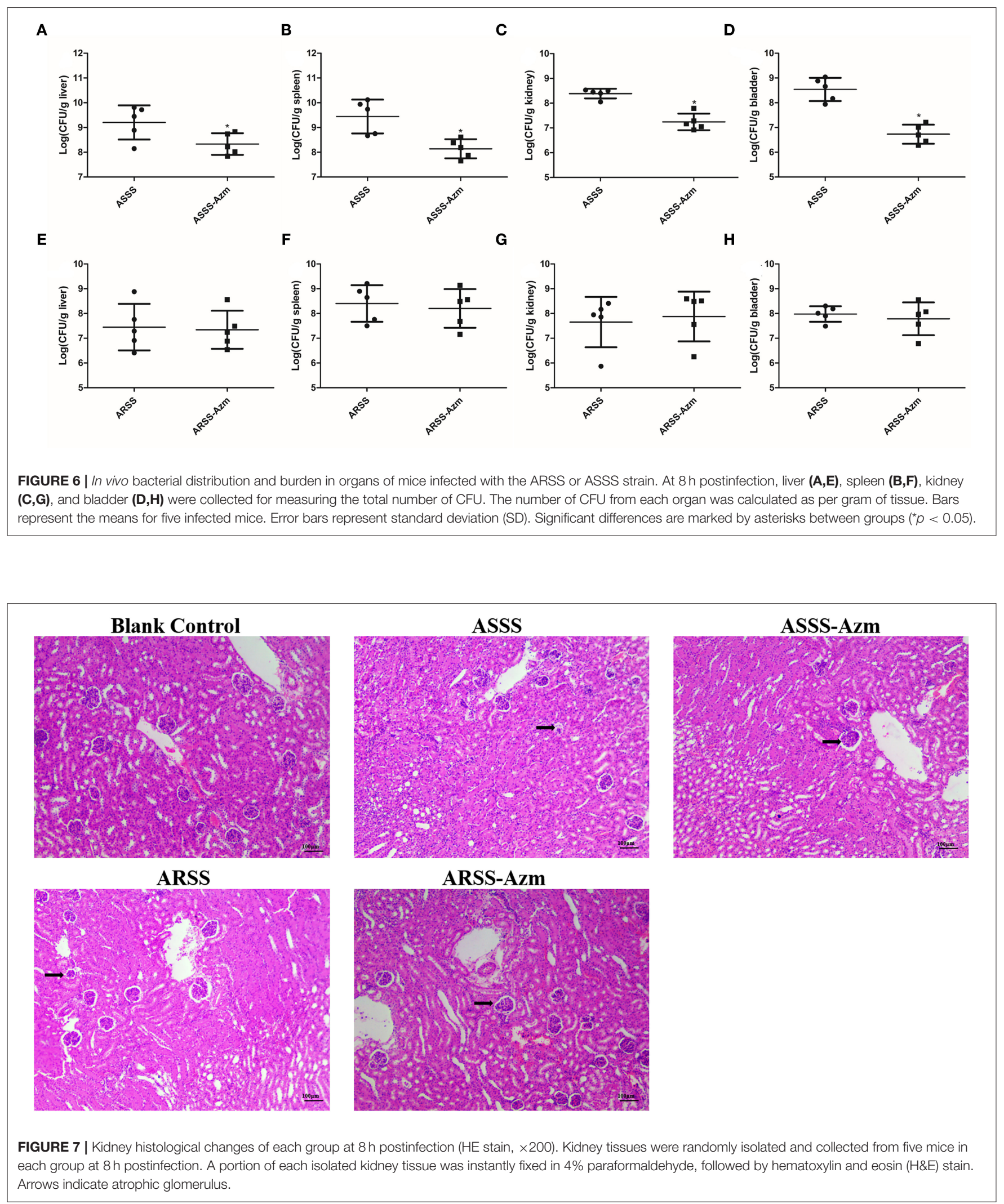

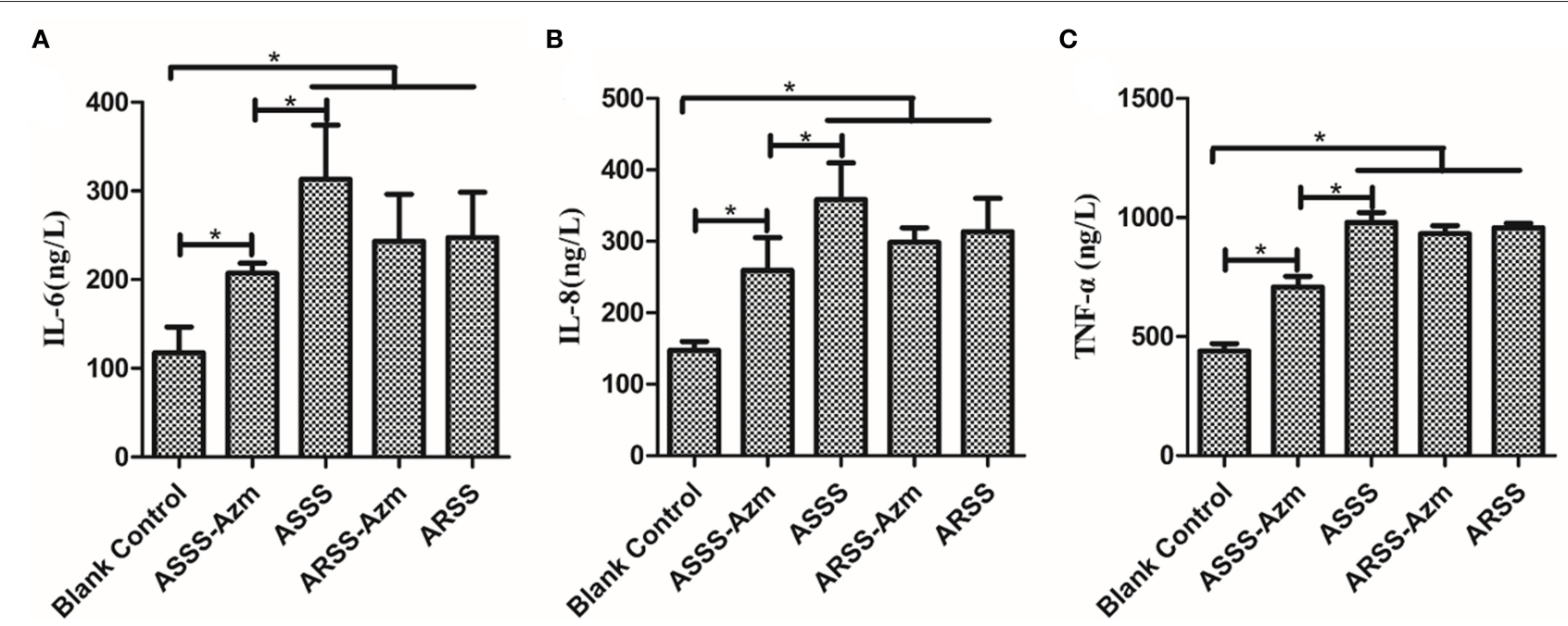

FIGURE 8 | Cytokine levels in the serum of mice i.p. injected with ASSS or ARSS. IL-6 (A), IL-8 (B), and TNF- $\alpha$ (C) levels in the serum were measured by ELISA at $8 \mathrm{~h}$ postinfection. Values were presented as the mean $\pm \mathrm{SD}$. Significant differences between groups are linked by brackets. ${ }^{\star} p<0.05$.

exposure to these compounds, and efflux pump inhibitors could reverse this resistance (20). Our previous investigation demonstrated that Bac could hinder the effect of MsrA efflux pump in ARSS by decreasing the ATP content and the mRNA transcript levels of the $m s r A$ gene (18). Simultaneously, ARSS recovered the sensitivity to Azm under the exposure of sub-MIC of Bac (Figure 2). The strain that recovered susceptibility was renamed ASSS. The mRNA transcript level of the msrA gene in the ASSS strain was lower than the ARSS strain (Figure 3A). What is important is that the rate of resistance reversal was highest in the $31.25-\mathrm{mg} / \mathrm{l}$ Bac group (Figure 2A). The reason for the phenomenon is that $62.5-250 \mathrm{mg} / \mathrm{l} \mathrm{Bac}$ moderately inhibited the growth of ARSS (Figure 1). The MICs of Azm against ARSS and ASSS were identical within 20 passages (Figure 2B). These results reflected that $\mathrm{Bac}$ is a potential antibiotic adjuvant of reversing resistance.

The major mechanisms of macrolide resistance to staphylococci involve (i) modification by methylation of 23S ribosomal rRNA, (ii) Msr family efflux pump upregulation, and (iii) macrolide inactivation by phosphotransferases or esterases (21). Also, biofilm is also the main factor influencing the efficiency of antibiotics. In this investigation, the ability of biofilm formation and the transcript levels of $m p h C$ and $m s r A$ efflux genes simultaneously decreased in the strain that recovered susceptibility to Azm (ASSS) compared with the ARSS strain (Figure 3). The expression of $m p h C$ in $S$. aureus was shown to be highly dependent on the presence of a portion of the gene encoding the MsrA efflux pump (22). It has been proposed that the transcriptional level of biofilm matrix components and biofilm formation could be decreased by genetic inactivation and inhibitor of efflux pumps (23). We previously indicated that Bac inhibited biofilm formation by modulating the MsrA efflux pump of ARSS
(18). Therefore, we surmised that along with the decrease of transcript levels of the $m s r A$ gene, the transcript level of the $m p h C$ gene and biofilm formation ability dwindled under the exposure of sub-MIC of Bac. The mechanisms needed to be further investigated.

At present, the therapeutics of anti-virulence have shown potential in preventing the bacteria from acquiring antibiotic resistance (24). It is reported that Bac suppressed the relative expression of virulence-related genes in S. aureus (25) and protected mice from lethal Shiga-like toxin 2 (Stx2) challenge by inducing Stx 2 to form inactive oligomers (26). In this study, serial passage of ARSS under sub-MIC of Bac over 20 days failed to produce resistant mutants (Table 2). Simultaneously, compared with the ARSS strain, the mRNA transcript levels of $d s d A$, ure $C$, Aas, uafA, and ssp genes were decreased in the ASSS strain (Figure 4). The activities of ureC and dsdA are essential for successful colonization and pathogenicity in S. saprophyticus (15). UafA of S. saprophyticus was a cell wall-anchored protein with an LPXTG motif (15). Proteins associated with the surface of S. saprophyticus (ssp) (1) and a fibronectin-binding autolysis Aas (16) were non-covalently surface-associated proteins. Therefore, we inferred that the colonization ability and pathogenicity of ASSS were lower than those of ARSS. When mice were infected with ARSS and ASSS in $\mathrm{OD}_{600 \mathrm{~nm}}$ of 2.71, the mortalities were 83.3 and $54.0 \%$, respectively (data not shown). Then we extended our study on the protective effects of Azm on the treatment of ASSS or ARSS infections in mice. In the verification experiment, Azm delayed the start time of death, alleviated the injury of the kidney, and decreased the bacterial burden in tissues and cytokine levels in mice infected with ASSS. In contrast, Azm could not treat the infection caused by the ARSS strain (Figures 5-8). These results suggested that Azm exhibited a better therapeutic efficacy on ASSS infection than ARSS infection. 


\section{CONCLUSION}

In conclusion, the sub-MIC of Bac reversed the resistance of ARSS to Azm. The strain that recovered sensitivity to Azm after being exposed to sub-MIC of Bac was named ASSS. The ability of biofilm formation and the mRNA transcript levels of $m s r A$, $m p h C$, and virulence genes were decreased in the ASSS strain. Azm successfully treated the infection caused by ASSS rather than ARSS. These results indicated that Bac could be expected to be developed into a new adjuvant of resistance reversal drug.

\section{DATA AVAILABILITY STATEMENT}

The original contributions presented in the study are included in the article/supplementary material, further inquiries can be directed to the corresponding author/s.

\section{ETHICS STATEMENT}

The animal study was reviewed and approved by Institutional Animal Care and Use Committee (IACUC) set by Nanjing Agricultural University (approval number: PTA030). Written

\section{REFERENCES}

1. Gatermann S, Kreft B, Marre R, Wanner G. Identification and characterization of a surface-associated protein (Ssp) of Staphylococcus saprophyticus. Infect Immun. (1992) 60:1055-60. doi: 10.1128/iai.60.3.1055-1060.1992

2. Wang J, Mingyu Q, Yefeng Z, Du H, Bai J, Yuan W, et al. In vitro synergistic effect of baicalin with azithromycin against Staphylococcus saprophyticus isolated from francolins with ophthalmia. Poultry Sci. (2019) 98:37380. doi: $10.3382 / \mathrm{ps} /$ pey356

3. Kim S, Moon DC, Park S, Kang HY, Na SH, Lim S. Antimicrobial resistance and genetic characterization of coagulase-negative staphylococci from bovine mastitis milk samples in Korea. J Dairy Sci. (2019) 102:1143948. doi: 10.3168/jds.2019-17028

4. Giguère S, Prescott JF, Dowling, PM. Antimicrobial Therapy in Veterinary Medicine, 4th ed. Blackwell publishing, Oxford, UK (2006).

5. Shrestha L, Bhattarai NR, Khanal B. Comparative evaluation of methods for the detection of biofilm formation in coagulase-negative staphylococci and correlation with antibiogram. Infect Drug Resist. (2018) 11:60713. doi: 10.2147/IDR.S159764

6. Feßler AT, Wang Y, Wu C, Schwarz S. Mobile macrolide resistance genes in staphylococci. Plasmid. (2018) 99:2-10. doi: 10.1016/j.plasmid.2018.05.001

7. Chajecka-Wierzchowska W, Zadernowska A, Nalepa B, Sierpińska M, Baniewska-Trokenheim A. Coagulase-negative staphylococci (CoNS) isolated from ready-to-eat food of animal origin-Phenotypic and genotypic antibiotic resistance. Food Microbiol. (2015) 46:222-6. doi: 10.1016/j.fm.2014.08.001

8. Kristiansen JE, Hendricks O, Delvin T, Butterworth TS, Aagaard L, Christensen JB, et al. Reversal of resistance in microorganisms by help of non-antibiotics. J Antimicrob Chemother. (2007) 59:1271-9. doi: 10.1093/jac/dkm071

9. Song M, Liu Y, Huang X, Ding S, Wang Y, Shen J, et al. A broad-spectrum antibiotic adjuvant reverses multidrug-resistant Gram-negative pathogens. Nat Microbiol. (2020) 5:1040-50. doi: 10.1038/s41564-020-0723-z

10. Cassel CK, Saunders RS. Engineering a better health care system: a report from the President's Council of Advisors on Science and Technology. JAMA. (2014) 312:787-8. doi: 10.1001/jama.2014.8906

11. Laxminarayan R. Antibiotic effectiveness: balancing conservation against innovation. Science. (2014) 345:1299-301. doi: 10.1126/science.1254163

12. Lynskey NN, Velarde JJ, Finn MB, Dove SL, Wessels MR. Roca binds csrs to modulate csrrs-mediated gene regulation in group a streptococcus. mBio. (2019) 10:e01495-19. doi: 10.1128/mBio.01495-19 informed consent was obtained from the owners for the participation of their animals in this study.

\section{AUTHOR CONTRIBUTIONS}

JW, JM, YW, and JL designed the experiment. JW, JZ, TQ, JD, and WW conducted the research. JW, ZL, and KL analyzed the data and wrote the manuscript. YW and DW revised the manuscript. The final manuscript has been read and approved by all authors.

\section{FUNDING}

This research was financially supported by the National Natural Science Foundation of China (NSFC, Grant No.31872514).

\section{ACKNOWLEDGMENTS}

We would like to thank all other people for their assistance with the experiments at the Institute of Traditional Chinese Veterinary Medicine of Nanjing Agricultural University.

13. Calvert MB, Jumde VR, Titz A. Pathoblockers or antivirulence drugs as a new option for the treatment of bacterial infections. Beilstein J Org Chem. (2018) 14:2607-17. doi: 10.3762/bjoc.14.239

14. Cho HS, Lee FH, Cho MH, Lee J. Red wines and flavonoids diminish Staphylococcus aureus virulence with anti-biofilm and anti-hemolytic activities. Biofouling. (2015) 31:1-11. doi: 10.1080/08927014.2014.99 1319

15. Kuroda M, Yamashita A, Hirakawa H, Kumano M, Morikawa K, Higashide $\mathrm{M}$, et al. Whole genome sequence of Staphylococcus saprophyticus reveals the pathogenesis of uncomplicated urinary tract infection. Proc Nat Acad Sci USA. (2005) 102:13272-7. doi: 10.1073/pnas.0502950102

16. Hell W, Meyer HW, Gatermann SG. Cloning of aas, a gene encoding a Staphylococcus saprophyticus surface protein with adhesive and autolytic properties. Mol Microbiol. (1998) 29:87181. doi: 10.1046/j.1365-2958.1998.00983.x

17. Novy P, Urban J, Leuner O, Vadlejch J, Kokoska L. In vitro synergistic effects of baicalin with oxytetracycline and tetracycline against Staphylococcus aureus. J Antimicrob Chemoth. (2011) 66:1298-300. doi: 10.1093/jac/ dkr108

18. Wang J, Jiao H, Meng J, Qiao M, Du H, He M, et al. Baicalin inhibits biofilm formation and the quorum-sensing system by regulating the MsrA drug efflux pump in Staphylococcus saprophyticus. Front Microbiol. (2019) 10:2800. doi: 10.3389/fmicb.2019.02800

19. Ming D, Wang D, Cao F, Xiang H, Mu D, Cao J, et al. Kaempferol inhibits the primary attachment phase of biofilm formation in Staphylococcus aureus. Front Microbiol. (2017) 8:2263. doi: 10.3389/fmicb.2017.02263

20. George AM, Levy SB. Amplifiable resistance to tetracycline, chloramphenicol, and other antibiotics in Escherichia coli: involvement of a non-plasmid-determined efflux of tetracycline. J Bacteriol. (1983) 155:531-40. doi: 10.1128/jb.155.2.531-540.1983

21. Fyfe C, Grossman TH, Kerstein K, Sutcliffe J. Resistance to macrolide antibiotics in public health pathogens. Csh Perspect Med. (2016) 6:a025395. doi: 10.1101/cshperspect.a025395

22. Matsuoka M, Inoue M, Endo Y, Nakajima Y. Characteristic expression of three genes, $\operatorname{msr}(\mathrm{A}), \mathrm{mph}(\mathrm{C})$ and $\operatorname{erm}(\mathrm{Y})$, that confer resistance to macrolide antibiotics onStaphylococcus aureus. FEMS Microbiol Lett. (2003) 220:28793. doi: 10.1016/S0378-1097(03)00134-4

23. Baugh S, Phillips CR, Ekanayaka AS, Piddock LJV, Webber MA. Inhibition of multidrug efflux as a strategy to prevent biofilm formation. J Antimicrob Chemoth. (2014) 69:673-81. doi: 10.1093/jac/dkt420 
24. Kong C, Neoh H, Nathan S. Targeting Staphylococcus aureus Toxins: a potential form of anti-virulence therapy. Toxins. (2016) 8:72. doi: $10.3390 /$ toxins 8030072

25. Zhang S, Hu B, Xu J, Ren Q, Wang Z, Wang S, et al. Baicalin suppress growth and virulence-related factors of methicillinresistant Staphylococcus aureus in vitro and vivo. Microb Pathogen. (2020) 139:103899. doi: 10.1016/j.micpath.2019.103 899

26. Dong J, Zhang Y, Chen Y, Niu X, Zhang Y, Yang C, et al. Baicalin inhibits the lethality of Shiga-like toxin 2 in mice. Antimicrob Agents Chemother. (2015) 59:7054-60. doi: 10.1128/AAC.01416-15

Conflict of Interest: The authors declare that the research was conducted in the absence of any commercial or financial relationships that could be construed as a potential conflict of interest.
Publisher's Note: All claims expressed in this article are solely those of the authors and do not necessarily represent those of their affiliated organizations, or those of the publisher, the editors and the reviewers. Any product that may be evaluated in this article, or claim that may be made by its manufacturer, is not guaranteed or endorsed by the publisher.

Copyright (C) 2022 Wang, Meng, Zhu, Qiu, Wang, Ding, Liu, Li, Wang, Liu and Wu. This is an open-access article distributed under the terms of the Creative Commons Attribution License (CC BY). The use, distribution or reproduction in other forums is permitted, provided the original author(s) and the copyright owner(s) are credited and that the original publication in this journal is cited, in accordance with accepted academic practice. No use, distribution or reproduction is permitted which does not comply with these terms. 